\title{
An Individual Differences Approach in Adaptive Waving of User Checkout Process in Retail eCommerce
}

\author{
Marios Belk ${ }^{1}$, Panagiotis Germanakos ${ }^{1,4}$, Stavros Asimakopoulos ${ }^{3}$, \\ Panayiotis Andreou ${ }^{1}$, Constantinos Mourlas ${ }^{3}$, George Spanoudis ${ }^{2}$, \\ and George Samaras ${ }^{1}$
}

${ }^{1}$ Department of Computer Science, University of Cyprus, CY-1678 Nicosia, Cyprus

\{belk, pgerman, panic, cssamara\} @cs . ucy.ac.cy

${ }^{2}$ Department of Psychology, University of Cyprus, CY-1678 Nicosia, Cyprus spanoud@ucy.ac.cy

${ }^{3}$ Faculty of Communication and Media Studies, National \& Kapodistrian University of Athens, 5 Stadiou Str, GR 105-62, Athens, Hellas

\{sasimako, mourlas\} @media.uoa.gr

${ }^{4}$ SAP AG, Dietmar-Hopp-Allee 16, 69190 Walldorf, Germany

\begin{abstract}
Developing a usable checkout process is pivotal for e-business success. However, recent research has shown that users frequently abandon their shopping carts and lacking a clear direction through the process. In this context, aiming to improve the usability and overall user experience of checkout processes in ecommerce Web-sites, this paper reports on a study, primarily inspired by concepts driven from theories of individual differences in cognitive processing, and considers content presentation and navigability as a measure of checkout usability and task quality. Concurrent think-aloud, short interviews and questionnaires were conducted with a convenient sample of 15 users to understand the preference of a particular type of checkout process, and users' task completion time while interacting with ecommerce Web-sites for a set of different checkout scenarios. Preliminary results revealed that cognitive styles have an effect on users' task completion and checkout process preference.
\end{abstract}

Keywords: Ecommerce, Checkout Process, Individual Differences, Cognitive Styles, User Study.

\section{$1 \quad$ Introduction}

Ensuring that checkout process design in an ecommerce retail environment is in alignment with the task at hand and providing satisfactory user experience (UX) is critical to business success. Meanwhile, user behaviours are changing profoundly; particularly due to the ways that technology is now being used as part of the shopping process. Researchers have devoted effort to develop metrics, guidelines and theories of Web-site and ecommerce usability, yet there still is a lack of consensus on the multi-faceted dimensions of checkout process as well as the relationships among user cognitive styles and their influence on checkout designs. The checkout process has 
become widely known over the years on the World Wide Web and making purchases online is a fairly standard process, with clear steps and expected outcomes [1]. Despite its popularity, research reveals that astonishingly between $60-70 \%$ of online users abandon shopping carts [2,3], while a recent study by [4], investigating the top 100 ecommerce checkout processes showed that $82 \%$ have usability issues. The authors in [2] argue that despite placing items in virtual shopping carts, online shoppers frequently abandon them; an issue that perplexes online retailers and has yet to be explained by scholars. Shopping cart abandonment occurs when a potential customer initiates an order by starting the checkout process, but exits the Web-site before the purchase is made. The most important reason for shopping cart abandonment appears to be that users are not given a clear direction through the process [4].

Studies on shopping cart abandonment mainly focused on the influence of psychological factors on this phenomenon [2, 5, 6, 7]. Moreover, several studies examined the relationship between navigational decisions and online purchase behavior $[8,9$, 10]. In this realm, taking into consideration that human-computer interactions during a checkout process are in principal cognitive tasks, we suggest that these interactions should be examined in more detail based on cognitive styles. Among numerous dimensions of individual differences in cognitive processing proposed in the literature [11], the work presented in this paper focuses on cognitive styles of the human mind as prior research has shown that cognitive styles of individuals affect user preference and performance in hypermedia systems [12]. Accordingly, the main research question is as follows: Do cognitive styles affect user preference and performance in different checkout process designs?

The remainder of the paper proceeds as follows: Section 2 briefly describes the relevant work on checkout process and theories of individual differences in cognitive styles. Section 3 describes the study methodology and results. Finally Section 4 concludes the paper.

\section{Related Work}

The World Wide Web has dramatically changed the way consumers purchase goods and services, collect information to compare products, and companies conduct their business. Recent statistics indicate that although there is a growing number of people that use the World Wide Web to search for product information, to do price comparisons, and to collect useful information in order to make their purchasing decision, the number of actual online purchases remains still relatively small [8, 13]. Although in the last years many scholars have provided frameworks and a number of methods to evaluate ecommerce Web-sites $[14,15]$, there is generally a lack of theoretical justifications of the frameworks and evaluation criteria they adopt. Moreover, these approaches do not effectively model the cognitive process of online users that determines how they perceive the quality of the Web-sites that are experiencing. Indeed, information processing and cognition are central activities when consumers interact with Web-sites [16]. 


\subsection{Checkout Processes}

Recent findings by industry analysts revealed that online consumers are impatient, easily dissatisfied and are likely to abandon their shopping carts and move to a different retailer if a Web-site's features fail to meet their expectations [17]. The work in [17] reports that attributes such as fast loading of pages, ease of navigation, efficient search and detailed product content are some of the features that online consumers expect from retail Web-sites and decrease the likelihood that consumers will leave sites without making purchases. The study in [6] found that besides immediate purchase intention, online shopping carts are also used for hedonic purposes such as securing price promotions, organizing items and as a 'wish list' for future purchases. This can be explained by the fact that, similarly to traditional retail shoppers, consumers shop online with utilitarian (e.g., goal-directed, task based) and/or hedonic (e.g., enjoyment gained by the shopping experience) motivations [18, 19]. Perceived risk related to privacy issues (e.g., sharing personal information with third parties), security aspects (e.g., non-delivery of products, transaction), and perceived waiting time (loading time), were found to influence shopping cart abandonment $[2,7]$. The study in [5] also found contextual factors (e.g., time pressure, uncertain need) and consumer characteristics (e.g., attitude toward online shopping) as factors influencing this phenomenon. The authors in [20] investigated the customization of the online purchase process of 422 electronic retailers relevant to the two constituent sub-processes in the online purchase process: i) decision customization; the customization of the information content delivered to users to help them in the decision-making sub-process; and ii) transaction customization; the customization of the purchase transaction subprocess for each user. The results indicated that decision customization that provides choice assistance by way of personalized product recommendations is positively associated with user satisfaction; and transaction customization, oriented towards making the transaction sub-process personal, convenient, and interactive is positively associated with user satisfaction with the purchase transaction sub-process. Additionally, the results indicate that both decision customization and transaction customization are associated with overall customer satisfaction with the online purchase process of electronic retailers. Recent research by [21] compared static displays with two rich media presentation formats (product videos and virtual product experience) and their impact on purchase intentions and willingness to pay in online stores. The results confirmed that the rich media displays enhanced the feeling of informedness about the examined products and increased excitement regarding the shopping experience. Virtual product experience had a direct positive effect on consumer purchase intentions, suggesting that virtual product experience-focused tools have the potential to outperform passive videos. Moreover, consumers showed higher willingness to pay values for experience products than for search products when interaction was possible.

\subsection{Individual Differences in Cognitive Styles}

Among numerous dimensions of individual differences proposed in the literature [22, 23, 24], the work presented in this paper focuses on cognitive styles. Research on cognitive styles is an area of human sciences that explains empirically observed differences in information mental representation and processing. Different theories have 
been proposed over time suggesting that individuals have differences in the way they process, remember, and recall information.

A widely accepted and accredited cognitive style dimension is considered the Wholist/Analyst cognitive style $[11,24]$ that refers to how individuals organize information and indicates a preference of structuring information as a whole to get the big picture (Wholists) or structuring the information in detail (Analysts). The main characteristics of each cognitive style are as follows:

Wholists: Users that belong to the Wholist type view a situation and organize information as a whole, proceed from analyzing the whole to its parts and organize information in loosely clustered wholes. Wholists have higher levels of assertiveness, and especially in extreme types, they are decisive in different situations.

Analysts: Users that belong to the Analyst type view a situation as a collection of parts, stress one or two aspects at a time, proceed form the parts to the whole and organize information in clear-cut groupings (chunking down). Analysts have low assertiveness and especially in extreme types, they are indecisive.

Several studies revealed that the Wholist/Analyst dimension has an effect on users' learning patterns and navigation behaviour within hypermedia systems [12, 26, 27]. A recent work in [12], which investigated the effect of cognitive styles on users' learning patterns within Web-instruction programs, revealed implications of cognitive style on users' preferred ways of using different navigation tools and display options. In particular, analyst users tended to actively group relevant concepts utilizing an alphabetical index tool of the hypermedia system, while Wholist users tended to be passive and relied on hierarchical maps to build relationships among different concepts [12]. A more recent study in [26] revealed a relationship between the Wholist/Analyst dimension and the users' navigation behaviour in terms of linearity/non-linearity. In particular, results revealed that Wholists tended to follow linear hyperlink sequences within online encyclopaedia articles, in contrast to Analysts who did not reveal any significant differences in navigation behaviour.

\section{Checkout Designs}

Based on the theoretical analysis, given that the Wholist/Analyst dimension is particularly related to the way hypermedia content is structured $[12,26]$, this paper aims to investigate the effect of the Wholist/Analyst dimension on user performance and preference of different content representation and navigation designs of checkout processes. We utilized different checkout designs of three existing ecommerce Web-sites: nordstrom.com (Nordstrom), discovery.com (Discovery), amazon.com (Amazon). The selection of the Web-sites was based on their different content presentation designs (i.e., textual or diagrammatical representation of content), and different navigation techniques (i.e., top-down checkout process or horizontal step-by-step checkout process). Figure 1 illustrates screenshots of each of the Web-sites. Table 1 presents the features of each Web-site based on the content presentation and navigation techniques used in the checkout process. 


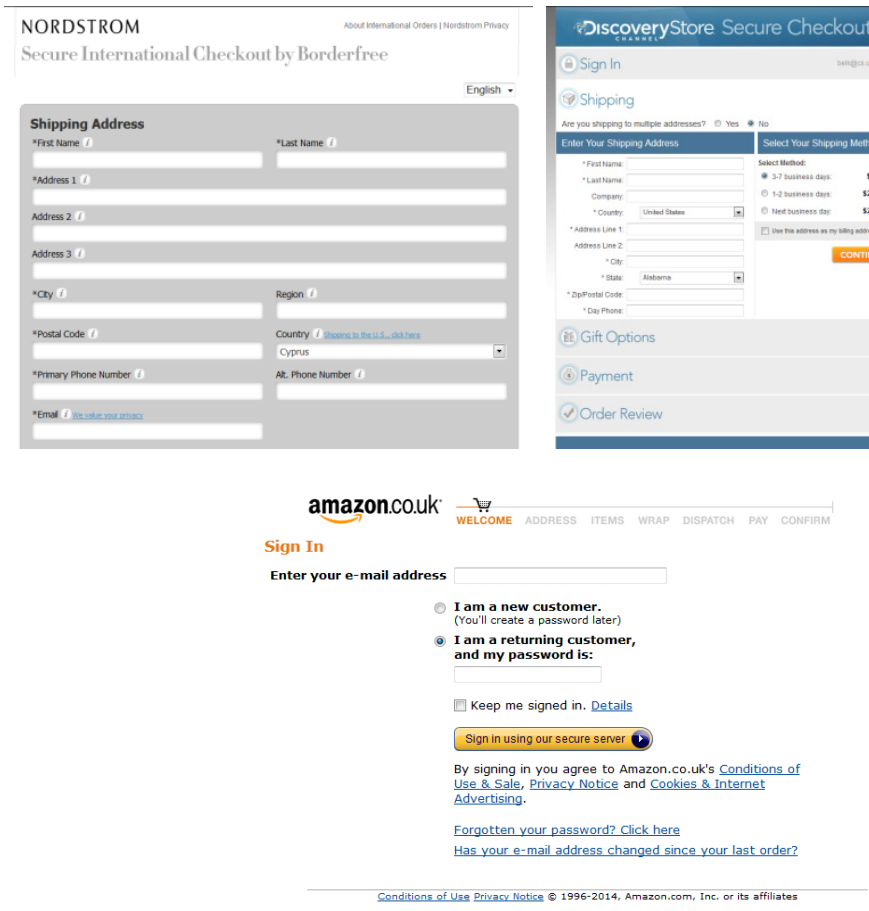

Fig. 1. Checkout process designs

Nordstrom illustrates content in a diagrammatical representation and follows a simple top-down navigation style in which users can freely enter the required information for performing the checkout process. All required information (shipping information, payment information, etc.) is visible in one single Web-page. This design has been selected since it freely enables users to access all steps of the checkout process in a single Web-page and could be related to the analytical approach that Analysts follow.

Table 1. Content presentation and navigation styles of each Web-site used in the study

\begin{tabular}{lcc}
\hline & Content Presentation & Navigation Technique \\
\hline $\begin{array}{l}\text { Nordstrom } \\
\text { Discovery }\end{array}$ & Graphical & Top-down with all steps visible \\
Amazon & Traphical & $\begin{array}{c}\text { Top-down step-by-step with only one } \\
\text { step active a time }\end{array}$ \\
& Textual & $\begin{array}{c}\text { Horizontal step-by-step with only one } \\
\text { step active at a time }\end{array}$ \\
\hline
\end{tabular}

Discovery similarly illustrates content in a diagrammatical representation and follows a guided top-down navigation style, with a single section being only active for entering the required information. In particular, users can only view and enter the required information for a single section (e.g., shipping information), and then need to 
submit the entered data in order to proceed to the next section. This design has been selected since it provides a top-down approach, guiding the user throughout all the steps of the checkout process and could be related to the wholistic, linear approach that Wholists follow.

Amazon illustrates content in a textual representation, without any additional graphical illustrations as in the other two Web-sites. A guided horizontal step-by-step navigation style is utilized for the checkout process in which users can only enter information of a particular section, and then proceed to the next section. A horizontal menu is utilized illustrating the current active section of the checkout process. Similarly to Discovery, the design of Amazon has been selected since it provides guidance throughout the checkout process that could be also related to the wholistic dimension of cognitive styles since it once more presents content through a constrained, guided environment. The main difference is that Amazon provides a horizontal guided approach and illustrates content in a clear textual representation, in contrast to the graphical representation of content in Discovery. It is important to mention that we intentionally selected two different content representations (textual vs. graphical) since we subsequently aimed to investigate whether the different type of content representation would affect the user interactions.

\section{$4 \quad$ Method of Study}

\subsection{Sampling and Procedure}

A total of 15 individuals (10 female and 5 male) participated voluntarily in a user study carried out during the first week of December 2013. All participants were undergraduate students and their age varied from 20 to 25 . The participants first completed a series of questions using a Web-based psychometric test based on Riding's CSA [25] that measured the response time on two types of cognitive tasks and computed the ratio between the response times for each task type in order to highlight differences in cognitive styles. Participants were required to compare whether two figures are identical, and whether one simple figure is part of another complex figure.

A within-subjects design was followed in which all participants navigated in three different ecommerce Web-sites; Nordstrom, Discovery and Amazon, for a set of different checkout scenarios. In each scenario, users were provided with a virtual credit card and assigned to select a product of their choice by adding it in their shopping basket and then execute the checkout process until buying the product. Given that users were interacting in a hypothetical, and not a real scenario of purchasing products, the think aloud protocol was conducted to investigate whether participants would complete or abandon the checkout process. In order to elicit users' subjective preference and perception of each Web-site checkout process, short interviews were conducted and questionnaires were provided at the end of each navigation scenario. Finally, the total time to complete the checkout process was measured to compare the usability of each checkout process in terms of efficiency. 


\subsection{Hypotheses}

The main hypothesis of this research is that cognitive styles affect task efficiency and user preference of checkout processes with different navigation styles. In particular, the following hypotheses were formulated for the purpose of our research.

$\boldsymbol{H}_{\boldsymbol{l}}$. Wholists are more efficient when interacting with designs that provide a step by step guided approach throughout the checkout process.

$\boldsymbol{H}_{2}$. Analysts are more efficient when interacting with non-constrained designs that enable full controllability of users during the checkout process.

$\boldsymbol{H}_{3}$. Cognitive styles affect user preference towards different design types of checkout process.

\subsection{Analysis and Results}

Results of the psychometric tests classified users as follows regarding their cognitive styles: 7 Wholists and 8 Analysts. The analysis investigates whether differences exist in task completion time and user preference toward a particular checkout design among users having different cognitive styles.

Task Efficiency. The total time to complete the checkout process for each design was recorded with the aim to investigate effects of cognitive styles on task efficiency of the different checkout designs. Table 2 illustrates the mean of time to complete the checkout process in each Web-site per cognitive style group. Results indicate that on average, Nordstrom had the most efficient checkout process. Based on the cognitive style groups, Analysts were more efficient in Nordstrom, whereas Wholists were more efficient in Amazon. Also, a comparison between the two cognitive style groups revealed that Analysts were more efficient in Nordstrom than Wholists, and Wholists were more efficient than Analysts in Amazon. Given that Wholists need more guidance in a hypermedia environment, the guided navigation style provided in Amazon has improved task efficiency of the checkout process. On the other hand, given that Analysts follow a more analytical approach during navigation and proceed from the parts to the whole, the fully-controllable and non-constrained checkout process of Nordstrom has positively affected task efficiency of that particular user group.

Table 2. Means of performances (in sec) for each checkout process and cognitive style group

\begin{tabular}{l|cc:c}
\hline & Wholists & Analysts & Overall \\
\hline Nordstrom & 117.07 & 99.88 & 107.9 \\
Discovery & 134.71 & 170.38 & 153.73 \\
Amazon & 116 & 130.88 & 123.93 \\
\hline
\end{tabular}

The results are very promising and indicate a tendency of verifying Hypothesis 1 and Hypothesis 2, however further studies need to be conducted with a larger sample to investigate main effects of cognitive styles on task efficiency in different designs of checkout processes. 
User Preference. In order to assess users' perceived preference and perception toward the different checkout processes, a five-item Likert-scale (1 Strongly Disagree 5 Strongly Agree) was designed that focused around perceived checkout preference, efficiency and effectiveness. Example questions were: "I completed the checkout process quickly", "I liked the design of the checkout process".

A tendency of Analysts preferring Nordstrom and Wholists preferring Amazon has been revealed (Figure 2). Based on the comments provided by the participants, the main reason of their preference was based on the particular navigation style followed in each checkout process. In particular, some Analysts that preferred Nordstrom reported that "I could access all information at once, without requiring navigating back and forth". On the other hand, Wholists that preferred Amazon reported that "I felt safe navigating through the steps with all the necessary information at hand". Another reason for the users' preference was based on the fact that users were already familiar and experienced using Amazon. Given that Analysts follow a scattered approach during navigation and need freedom of operation, the design of Nordstrom has positively affected their preference, compared to Wholists who preferred Amazon since this user group needs more guidance during navigation.

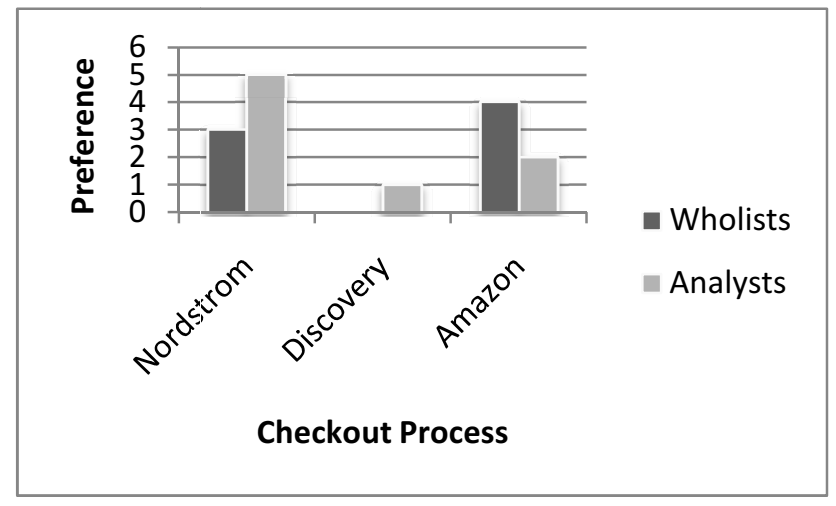

Fig. 2. Preference per cognitive style group

Finally, when asked with which checkout process they felt more secure, and whether users would abandon the checkout process in a real-life scenario, the majority of Wholist users reported that they would most probably abandon the Nordstrom checkout process compared to the other Web-sites. The main reason was that they didn't like the overall "look-and-feel" of the checkout process, and mainly because no guidance or any status about the process was provided by the Web-site during interaction. Regarding the Discovery checkout process, the majority of users across all user groups would abandon the checkout process. As participants responded, the main reason is that "I didn't like the design" (Analyst), "I didn't know what the next step was" (Wholist), "There was too much content illustrated" (Wholist). In contrast, in the case of Amazon, the majority felt secure during interaction and would not abandon the checkout process. Based on responses, this might be based on the familiarity factor of Amazon being a popular and accredited ecommerce Web-site. 


\section{Conclusions}

Checkout is an important stage of online purchasing; thus allowing users more efficient decision-making and supporting their information processing and cognitive ability is of critical importance. In this realm, aiming to understand users during interactions within checkout processes, a user study was conducted that explored the impact of the Wholist-Analyst cognitive style to evaluate checkout process from the user viewpoint in a retail ecommerce setting. Preliminary results based on quantitative and qualitative measures revealed that cognitive styles affect users' task completion and checkout process preference. In particular, Wholist users have shown a preference toward checkout processes following a more guided approach, as in the case of Amazon. Quantitative measures obtained have shown that Wholist users were also more efficient during interaction in Amazon. Similarly, Analyst users were more efficient and preferred Nordstrom which did not follow a guided approach and provided more freedom to users for entering their information. Such a result suggests that designers and developers should bear in mind individual differences in cognitive styles when designing checkout processes. A practical implication of this result would be based on a personalization engine for providing the "best-fit" navigation style (non-constrained or guided) in a checkout process, considering the users' cognitive styles.

Findings of this study are expected to provide useful insights for practitioners to develop more usable checkout processes and for researchers to better assess the effect of user cognitive styles on online checkout process behavior. In future work we plan to further extend our study with a larger sample and different checkout process designs as well as utilize different cognitive styles such as the Verbal/Imager dimension [11], and more basic cognitive processes such as Working Memory.

Acknowledgements. The work is co-funded by the PersonaWeb project under the Cyprus Research Promotion Foundation (ТПЕ/П $\Lambda$ HРО/0311(BIE)/10), and the EU project SocialRobot (285870).

\section{References}

1. Nielsen, J.: Ecommerce Usability, http: / / www . nngroup.com/articles / e-commerce-usability (retrieved October 12, 2013)

2. Kukar-Kinney, M., Close, A.G.: The Determinants of Consumers' Online Shopping Cart Abandonment. Journal of the Academy of Marketing Science 38(2), 240-250 (2010)

3. Close, G.A., Kukar-Kinney, M.: Beyond Buying: Motivations Behind Consumers' Online Shopping Cart Use. Journal of Business Research 63, 986-992 (2010)

4. Appleseed, J., Holst, C.: E-Commerce Checkout Usability: Exploring the Customer's Checkout Experience. Baymard Institute, Copenhagen (2013)

5. Cho, C.H., Kang, J., Cheon, H.J.: Online shopping hesitation. Cyber Psychology and Behavior 9(3), 261-274 (2006)

6. Moore, S., Matthews, S.: An exploration of the online shopping cart abandonment syndrome: A matter of risk and reputation. Journal of Website Promotion 2, 71-88 (2006) 
7. Rajamma, R.K., Paswan, A.K., Hossain, M.M.: Why do shoppers abandon shopping cart? Perceived waiting time, risk, and transaction inconvenience. Journal of Product and Brand Management 18, 188-197 (2009)

8. Moe, W.W., Fader, P.S.: Capturing evolving visit behavior in clickstream data. Journal of Interactive Marketing 18(1), 5e-19e (2004)

9. Sismeiro, C., Bucklin, R.E.: Modeling purchase behavior at an E-commerce web site: A task-completion approach. Journal of Marketing Research 41, 306-323 (2004)

10. Van den Poel, D., Buckinx, W.: Predicting Online Purchasing Behaviour. European Journal of Operational Research 166(2), 557-575 (2005)

11. Riding, R., Cheema, I.: Cognitive Styles - An Overview and Integration. Journal of Educational Psychology 11(3-4), 193-215 (1991)

12. Chen, S., Liu, X.: An Integrated Approach for Modeling Learning Patterns of Students in Web-Based Instruction: A Cognitive Style Perspective. ACM Transactions on ComputerHuman Interaction 15(1), 1-28 (2008)

13. Storto, C.: Evaluating ecommerce websites cognitive efficiency: An integrative framework based on data envelopment analysis, Applied Ergonomics 44, 1004e-1014e (2013)

14. Boyd, A.: The goals, questions, indicators, measures (GQIM) approach to the measurement of customer satisfaction with e-commerce web sites. Aslib 54(3), 177e-187e (2002)

15. Merwe, R., Bekker, J.: A framework and methodology for evaluating e-commerce web sites. Internet Research: Electronic Networking Applications and Policy 13(5), 330-341 (2003)

16. Zhang, P., von Dran, G.M.: Satisfactor and dissatisfactors: a two-factor model for website design and evaluation. Journal of the American Society for Information Science 51(4), $1253 \mathrm{e}-1268 \mathrm{e}(2000)$

17. Forrester Consulting: eCommerce Web Site Performance Today: An Updated Look At Consumer Reaction To A Poor Online Shopping Experience (2009)

18. Arnold, M., Reynolds, K.: Hedonic shopping motivations. Journal of Retailing 79(2), 77-95 (2003)

19. Bridges, E., Florsheim, R.: Hedonic and utilitarian shopping goals: the online experience. Journal of Business Research 61(4), 309-314 (2008)

20. Thirumalai, S., Sinha, K.K.: Customization of the online purchase process in electronic retailing and customer satisfaction: An online field study. Journal of Operations Management 29, 477-487 (2011)

21. Li, T., Meshkova, Z.: Examining the impact of rich media on consumer willingness to pay in online stores. Electronic Commerce Research and Applications 12(6), 449-461 (2013)

22. Sternberg, R.J.: Thinking Styles. Cambridge University Press, New York (1997)

23. Witkin, H.A., Moore, C.A., Goodenough, D.R., Cox, P.W.: Field-dependent and Dieldindependent Cognitive Styles and their Educational Implications. J. Review of Educational Research 47(1), 1-64 (1977)

24. Peterson, E., Deary, I., Austin, E.: A New Reliable Measure of Verbal-Imagery Cognitive Style. Personality and Individual Differences 38, 1269-1281 (2005)

25. Riding, R.: Cognitive Styles Analysis. Learning and Training Technology, Birmingham, U.K (2001)

26. Belk, M., Papatheocharous, E., Germanakos, P., Samaras, G.: Modeling Users on the World Wide Web based on Cognitive Factors, Navigation Behaviour and Clustering Techniques. J. Systems and Software 86(12), 2995-3012 (2013)

27. Germanakos, P., Tsianos, N., Lekkas, Z., Mourlas, C., Samaras, G.: Realizing Comprehensive User Profile as the Core Element of Adaptive and Personalized Communication Environments and Systems. J. The Computer Journal 52(7), 749-770 (2008) 